\title{
OBSERVATÓRIO SOCIOAMBIENTAL EM SEGURANÇA ALIMENTAR E NUTRICIONAL: ANÁLISE DOS INDICADORES DE PRODUÇÃO DE ALIMENTOS EM NÍVEL MUNICIPAL NO RIO GRANDE DO SUL
}

\section{SOCIO-ENVIRONMENTAL FOOD SECURITY OBSERVATORY: ANALYSIS OF FOOD PRODUCTION INDICATORS AT THE MUNICIPAL LEVEL IN RIO GRANDE DO SUL}

Angélica Cristina da Siqueira

Universidade Federal do Rio Grande do Sul - Porto Alegre - RS - Brasil

\section{Daniela Wives}

Universidade Federal do Rio Grande do Sul - Porto Alegre - RS - Brasil

Tathiane Muriel Medeiros

Universidade Federal do Rio Grande do Sul - Porto Alegre - RS - Brasil

Natany Meregalli Schreiber

Universidade Federal do Rio Grande do Sul - Porto Alegre - RS - Brasil

Leonardo Xavier da Silva

Universidade Federal do Rio Grande do Sul - Porto Alegre - RS - Brasil

\section{Gabriela Coelho-de-Souza}

Universidade Federal do Rio Grande do Sul - Porto Alegre - RS - Brasil

Resumo: Desde a década de 1990, a temática dos Direitos Humanos e da Segurança Alimentar e Nutricional - SAN vem sendo desenvolvida no Brasil, culminando na construção da Política e do Plano Nacional de SAN (PNSAN e PLANSAN 2012/2015). O PLANSAN propõe 60 indicadores divididos em sete dimensões, os quais estabelecem clara relação com os Objetivos de Desenvolvimento do Milênio. Com o intuito de operacionalizar esses indicadores no Estado do Rio Grande do Sul - RS foi criado o Observatório Socioambiental em Segurança Alimentar e Nutricional do Rio Grande do 
Sul - OBSSAN-RS, em parceria com o Conselho Estadual de Segurança Alimentar e Nutricional - CONSEA-RS e o Departamento de Informática da UFRGS. Neste contexto, o objetivo deste trabalho é apresentar a análise dos indicadores da Dimensão I (Produção de Alimentos) do OBSSAN-RS em nível municipal, discutindo a importância dessa ferramenta para o monitoramento do estabelecimento do Direito Humano à Alimentação Adequada - DHAA. Os dados disponíveis para o nível municipal foram comparados com os indicadores sugeridos pelo PLANSAN. Observou-se que, apesar de contemplarem informações importantes para a compreensão do contexto da produção de alimentos dos municípios do estado, os indicadores propostos alcançam apenas em parte as necessidades para o monitoramento da SAN neste nível territorial, sendo necessária sua adaptação para a avaliação da SAN nos municípios do RS.

Palavras-chave: Segurança Alimentar e Nutricional. Indicadores. Plano Nacional de Segurança Alimentar e Nutricional. Rio Grande do Sul.

Abstract: Since the 1990, the theme of Human Rights and Food Security (FS) has been developed in Brazil, culminating in the construction of the National Policy and Plan of Food Security (PNSAN and PLANSAN 2012/2015). The PLANSAN proposes sixty indicators divided into seven dimensions, which are clearly related to the Millennium Development Goals. In order to operationalize these indicators in the State of Rio Grande do Sul (RS), the Socio-Environmental Food Security Observatory of Rio Grande do Sul (OBSSAN-RS) was created in partnership with the State Council for Nutrition and Food Security (CONSEA-RS) and the Informatics Department of UFRGS. In this context, the aim of this paper is to present the analysis of indicators of Dimension I (Food Production) of the OBSSAN-RS at municipal level, discussing the importance of this tool for monitoring the establishment of the Human Right to Adequate Food (DHAA). The data available to the municipal level were compared with the indicators suggested by PLANSAN. It was observed that although contemplate important information for understanding the context of food production in the municipalities of the state, the proposed indicators have not reached entirely the requirements to monitoring the SAN in this territorial level, so adapting it is necessary to rating the state of SAN in municipalities of the RS.

Keywords: Food Security. National Plan for Food Security. Indicators. Rio Grande do Sul.

\section{Introdução}

O debate sobre os Direitos Humanos vem se fortalecendo desde a Declaração Universal dos Diretos Humanos, de 1948. No Brasil, ele se consolida a partir da Constituição Federal de 1988, com a denominada Constituição Cidadã. Os Direitos Humanos estão agrupados em doze categorias, a saber: Direito à Vida; à Liberdade e Segurança da Pessoa; à Satisfação do Mais Alto Padrão de Saúde Física e Mental; de não ser Submetido a Tortura, Tratamento e Punição Desumanos ou Degradantes; de participar de Manifestações Públicas; à Educação; à 
Moradia Adequada; à Seguridade Social; ao Trabalho; à Liberdade de Expressão e Opinião; a Julgamento Justo; e à Alimentação Adequada. Todos são Direitos universais, inalienáveis, inter-relacionados, interdependentes, iguais e não discriminatórios.

De acordo com Teles et al (2011), atualmente há uma carência de medidas que forneçam mensuração apropriada dos Direitos Humanos, não apenas das violações de direitos, mas também de onde, quanto e como o país tem avançado na realização dos Direitos Humanos. Dentre os Direitos Humanos, o Direito à Alimentação Adequada está intimamente associado à SAN. No Brasil, essa temática vem sendo desenvolvida desde a década de 1990, culminando em um processo de mobilização social entre sociedade civil e governo federal, que resultou na criação do Sistema Nacional de Segurança Alimentar e Nutricional SISAN, através da, Lei Orgânica de Segurança Alimentar Nutricional LOSAN - Lei $n^{\circ} 11.346 / 2006$. O conceito de SAN alterou-se no decorrer dos anos, ampliando-se especialmente no que se refere a questões socioeconômicas e culturais, levando-se em conta o contexto no qual os sujeitos de direitos estão inseridos, e reconhecendo a relevância dos saberes e práticas na construção da segurança alimentar e nutricional.

A IV Conferência Nacional de SAN destacou a importância do direcionamento de políticas públicas de SAN para povos indígenas e povos e comunidades tradicionais de acordo com suas tradições culturais e alimentares (CONSEA, 2011). Essa pauta ganhou ainda mais força na agenda pública pelo forte diálogo com a Política Nacional de Gestão Ambiental e Territorial em Terras Indígenas (BRASIL, 2012), e a Política Nacional de Desenvolvimento Sustentável dos Povos e Comunidades Tradicionais (BRASIL, 2007).

Nesse contexto, foram estabelecidas as bases para a construção da Política e do Plano Nacional de SAN (PNSAN e PLANSAN 2012/2015), institucionalizados pelo Decreto Federal 7.272/2010. O Plano Nacional de SAN apresenta os indicadores propostos para o monitoramento da implementação das políticas em SAN no Brasil. De acordo com o Alto Comissariado da Organização das Nações Unidas, indicadores são informações específicas quanto ao estado de um evento, atividade ou resultado relacionado a normas e padrões de Direitos Humanos, o qual focaliza e reflete preocupações e princípios de Direitos Humanos e permite a avaliação e o monitoramento da promoção e proteção dos 
mesmos. Os indicadores devem refletir as obrigações do Estado de respeitar, proteger e realizar os Direitos Humanos, além de medir o esforço do Estado para o cumprimento (Teles et al, 2011).

O PLANSAN propõe 60 indicadores divididos em sete dimensões, quais sejam: 1) Produção de alimentos; 2) Disponibilidade de Alimentos; 3) Renda e Condições de Vida; 4) Acesso à alimentação adequada e saudável, incluindo água; 5) Saúde, Nutrição e Serviços Relacionados; 6) Educação; e 7) Programas e Ações de Segurança Alimentar e Nutricional. Percebe-se relação com os Objetivos de Desenvolvimento do Milênio propostos pelos 189 Estados Membros das Nações Unidas no ano de 2000: 1. Erradicar a pobreza extrema e a fome; 2. Alcançar o ensino primário universal; 3. Promover a igualdade de gênero e a autonomização da mulher; 4. Reduzir a mortalidade de crianças; 5. Melhorar a saúde materna; 6. Combater o VIH/SIDA, a malária e outras doenças; 7. Garantir a sustentabilidade ambiental; e 8. Criar uma parceria global para o Desenvolvimento (Fundo das Nações Unidas para a Infância - UNICEF, 2014).

Com o intuito de operacionalizar o monitoramento desses indicadores no Estado do Rio Grande do Sul foi criado o OBSSAN-RS pelo Núcleo de Estudos e Pesquisas em Segurança Alimentar e Nutricional, vinculado ao Programa de Pós-Graduação em Desenvolvimento Rural PGDR, em parceria com o CONSEA-RS e o Departamento de Informática da UFRGS.

O OBSSAN-RS se propõe a atuar como espaço de estudo, pesquisa, e extensão, desenvolvendo um espaço de interlocução institucional e estabelecendo um fluxo de informações, em nível estadual e municipal, por meio de um software (http://www.ufrgs.br/pgdr/nesan/obssan.htm) que disponibiliza o banco de dados de acesso público com informações sistematizadas sobre as dimensões propostas no PLANSAN 2012/2015. Além das dimensões propostas pelo PLANSAN, o OBSSAN está desenvolvendo a dimensão "Povos indígenas e comunidades tradicionais", buscando dar visibilidade às condições de vida desses grupos nos diferentes municípios.

Recentemente, os Observatórios de Políticas Públicas vêm adquirindo grande relevância no país, a partir do fortalecimento das Políticas Públicas geridas junto ao sistema de democracia 
representativa. Seu papel almeja contribuir para a transparência de informações de interesse público, subsidiando a participação e o controle social; como também, em produzir análises alternativas às versões do Governo, em parceria com atores sociais das comunidades e instituições da área, gerando novos conhecimentos, não apenas informação. Nesse sentido, um grande desafio que se apresenta relaciona-se à construção ou operacionalização de instrumentos de avaliação de políticas públicas. Buscando operacionalizar o instrumento criado no PLANSAN, para viabilizar o acompanhamento da efetividade das políticas de segurança alimentar e nutricional, um primeiro passo é analisar a pertinência das dimensões e indicadores propostos. Dessa forma, o objetivo deste trabalho é analisar os indicadores da primeira dimensão - Produção de Alimentos - do OBSSAN-RS em nível municipal no Rio Grande do Sul, discutindo a importância dessa ferramenta para o monitoramento do estabelecimento do Direito Humano à Alimentação Adequada.

Este trabalho está dividido em cinco momentos. Primeiramente, busca-se realizar uma breve contextualização, problematização e apresentar o objetivo central do estudo. Após a introdução, apresentase o caminho metodológico percorrido para viabilizar elaboração. 0 terceiro e o quarto momento apresentam e discutem os resultados. Finalizando, são levantadas as considerações finais.

\section{Procedimentos Metodológicos}

Tendo em vista o caráter do objeto de estudo, o modo de pesquisa utilizado é o exploratório, entendendo a necessidade, primeira, de explicitá-lo e/ ou construir hipóteses. Conforme Gerhadt e Silveira (2009) a grande maioria dessas pesquisas envolve um levantamento bibliográfico ou entrevistas com pessoas que tiveram experiências práticas com o problema pesquisado. Também utiliza análise de exemplos que contribuam para a compreensão do objeto em foco. Quanto aos procedimentos, o referido estudo empregou pesquisa documental.

O projeto em andamento caracteriza-se por uma inter-relação entre pesquisa, extensão e ensino, envolvendo três etapas. A primeira consiste na pesquisa de dados em fontes secundárias. A segunda etapa 
contempla a inserção dos dados em plataforma da web desenvolvida em parceria com o Departamento de Informática. Por fim, paralelamente, são fomentadas atividades de ensino e extensão na Universidade, alimentando os bancos de dados do OBSSAN-RS, monitorando o trabalho realizado, e mantendo a comunicação entre sociedade e instituições em um processo dialógico sobre a PNSAN, pela pesquisaação-participativa.

\subsection{Seleção dos dados da Dimensão I}

Buscando refletir sobre os indicadores de monitoramento sugeridos pela Câmara Interministerial de Segurança Alimentar e Nutricional - CAISAN, foram selecionados dados do Censo Agropecuário (2006), da Pesquisa Agrícola Municipal (2000 - 2011) e da Pesquisa Municipal (2000 - 2011) do Instituto Brasileiro de Geografia e Estatística - IBGE, em nível municipal e também agregados nas micro e mesorregiões do Estado do Rio Grande do Sul. Os dados foram definidos a partir de avaliação das tabelas disponibilizadas nos bancos de dados supracitados, contemplando todas as que possuem informações relacionadas aos indicadores desta Dimensão:

a) Indicador 1 - Percentual da produção da agricultura familiar na produção total de alimentos (por produtos);

b) Indicador 2 - Percentual da área ocupada pela agricultura familiar em relação à área total de produção;

c) Indicador 3 - Percentual da área ocupada por grupo dos principais produtos dirigidos para alimentação no mercado interno em relação à área total;

d) Indicador 4 - Percentual da área sob utilização de sistemas sustentáveis de produção (Agricultura Orgânica + Plantio Direto)

\subsection{Coleta e sistematização de dados}

Os dados são coletados diretamente da plataforma virtual do sistema IBGE de recuperação automática - SIDRA, no formato tabela. Posteriormente, é realizada a sistematização em uma tabela padrão pelo software OpenOffice.org Calc 3.4.1, na qual os dados são organizados por anos, códigos e nomes dos locais (país, estado, micro e 
mesorregiões, e municípios), conforme classificações determinadas pelo IBGE.

Ao final da coleta e sistematização dos dados de cada indicador, é realizada auditoria para avaliar a manutenção da fidedignidade dos dados após a reorganização. Para tal, são selecionados, aleatoriamente, $25 \%$ do total de municípios e comparados os dados com a fonte original.

Os dados já auditados serão importados para sistema específico que está sendo desenvolvido em parceria com o Departamento de Informática da UFRGS. Esse processo é realizado por meio de reuniões periódicas entre os pesquisadores do OBSSAN-RS e a equipe do Departamento de Informática. O sistema possibilitará a visualização dos dados em um mapa do Rio Grande do Sul, além da exportação em formato de tabela e gráficos.

\subsection{Análise dos dados e indicadores}

Os dados disponíveis para nível municipal foram comparados com os indicadores sugeridos pela CAISAN, avaliando as possibilidades de agregação das informações para responder a cada indicador.

Alguns dados não podem ser agregados, ou não correspondem exatamente aos indicadores propostos no PLANSAN 2012/2015, porém não foram excluídos do banco de dados do OBSSAN-RS por entender-se que podem contribuir para a avaliação da SAN dos municípios.

\section{Detalhamentos dos indicadores da Dimensão 1 do PLANSAN}

Nas tabelas abaixo, constam os dados selecionados para cada Indicador proposto pelo CAISAN, disponíveis nos bancos de dados na escala municipal, regional e estadual. Todos os dados selecionados para os Indicadores 1, 2 e 3 já foram coletados, sistematizados e auditados, com exceção dos dados de produtos da horticultura referente ao Indicador 1 (em destaque na Tabela 1), que ainda estão em fase de definição dos produtos a serem considerados na coleta de dados. Todos os dados para o Indicador 4 ainda estão em fase de definição e coleta. 
3.1 Indicador 1 - Percentual da produção da agricultura familiar na produção total de alimentos (por produtos)

Foram selecionados os dados citados no Quadro 01, visando refletir a produção de alimentos no estado.

Vale destacar que apenas os dados coletados a partir do Censo Agropecuário poderão compor o cálculo de percentual da produção da agricultura familiar na produção total de alimentos, visto que, nas demais fontes consultadas, as pesquisas não consideram diferenciação do tipo de agricultura praticada na produção.

Dessa forma, tendo em vista que os dados do Censo Agropecuário referem-se apenas a produtos de origem animal, e alguns gêneros da horticultura, os demais produtos não poderão ser agregados para análise conforme indicado pela CAISAN. Porém, ainda assim foram incluídos no banco de dados do OBSSAN-RS, pois compõem a produção agrícola do estado e, portanto, refletem informações importantes para a análise da Segurança Alimentar e Nutricional dos municípios do Rio Grande do Sul.

Ainda ressalta-se que foram incluídos dados de números de estabelecimentos agropecuários e de pessoas ocupadas nos estabelecimentos de agricultura da agricultura familiar e não-familiar, visando obter informações mais profundas para a caracterização do cenário de produção de alimentos dos municípios.

Quadro 01. Dados selecionados para o Indicador 1, da Dimensão I

\begin{tabular}{|l|l|l|}
\hline \multicolumn{1}{|c|}{ Fonte } & \multicolumn{1}{|c|}{ Dados selecionados } & \multicolumn{1}{|c|}{$\begin{array}{c}\text { Variável } \\
\text { selecionada }\end{array}$} \\
\hline $\begin{array}{l}\text { Censo } \\
\text { Agropecuário }\end{array}$ & $\begin{array}{l}\text { Estabelecimentos agropecuários com aves na agricultura } \\
\text { familiar, não familiar e total }\end{array}$ & $\begin{array}{l}\text { Número de } \\
\text { cabeças de aves }\end{array}$ \\
\hline $\begin{array}{l}\text { Censo } \\
\text { Agropecuário }\end{array}$ & $\begin{array}{l}\text { Estabelecimentos agropecuários com suínos na agricultura } \\
\text { familiar, não familiar e total }\end{array}$ & $\begin{array}{l}\text { Número de } \\
\text { cabeças de } \\
\text { suínos }\end{array}$ \\
\hline $\begin{array}{l}\text { Censo } \\
\text { Agropecuário }\end{array}$ & $\begin{array}{l}\text { Estabelecimentos agropecuários com bovinos na agricultura } \\
\text { familiar, não familiar e total }\end{array}$ & $\begin{array}{l}\text { Número de } \\
\text { cabeças de } \\
\text { bovinos }\end{array}$ \\
\hline $\begin{array}{l}\text { Censo } \\
\text { Agropecuário }\end{array}$ & $\begin{array}{l}\text { Estabelecimentos agropecuários com vacas ordenhadas e } \\
\text { leite produzido na agricultura familiar, não familiar e total }\end{array}$ & $\begin{array}{l}\text { Número de } \\
\text { cabeças de vacas } \\
\text { ordenhadas, e }\end{array}$ \\
\hline
\end{tabular}


Angélica C. Siqueira, Daniela Wives, Tathiane M. Medeiros, Natany M. Schreiber, Leonardo X. Silva, Gabriela C. Souza

\begin{tabular}{|l|l|l|}
\hline & & litros de leite \\
\hline $\begin{array}{l}\text { Censo } \\
\text { Agropecuário }\end{array}$ & $\begin{array}{l}\text { Estabelecimentos agropecuários na agricultura familiar, não } \\
\text { familiar e total }\end{array}$ & $\begin{array}{l}\text { Número de } \\
\text { estabelecimento } \\
\text { s agropecuários }\end{array}$ \\
\hline $\begin{array}{l}\text { Censo } \\
\text { Agropecuário }\end{array}$ & $\begin{array}{l}\text { Estabelecimentos agropecuários com suínos na agricultura } \\
\text { familiar, não familiar e total }\end{array}$ & $\begin{array}{l}\text { Número de } \\
\text { estabelecimento } \\
\text { s agropecuários }\end{array}$ \\
\hline $\begin{array}{l}\text { Censo } \\
\text { Agropecuário }\end{array}$ & $\begin{array}{l}\text { Pessoal ocupado com e sem laço familia na agricultura } \\
\text { familiar, não familiar e total }\end{array}$ & $\begin{array}{l}\text { Número de } \\
\text { pessoas } \\
\text { ocupadas }\end{array}$ \\
\hline
\end{tabular}


Continuação

\begin{tabular}{|c|c|c|}
\hline Fonte & Dados selecionados & $\begin{array}{l}\text { Variável } \\
\text { selecionada }\end{array}$ \\
\hline $\begin{array}{l}\text { Pesquisa } \\
\text { Pecuniária } \\
\text { Municipal }\end{array}$ & $\begin{array}{l}\text { Rebanhos de Bovinos, Equinos, Bubalinos, Asininos, Muares, } \\
\text { Suínos, Caprinos, Ovinos, Galos, frangas, frangos e pintos, } \\
\text { Galinhas, Codornas, Coelhos }\end{array}$ & $\begin{array}{l}\text { Número de } \\
\text { cabeças }\end{array}$ \\
\hline $\begin{array}{l}\text { Pesquisa } \\
\text { Pecuniária } \\
\text { Municipal }\end{array}$ & $\begin{array}{l}\text { Produção de Coelhos, Leite (Mil litros), Ovos de galinha (Mil } \\
\text { dúzias), Ovos de codorna (Mil dúzias), Mel de abelha } \\
\text { (Quilogramas) }\end{array}$ & $\begin{array}{l}\text { Quantidade } \\
\text { produzida de } \\
\text { gêneros } \\
\text { alimentares }\end{array}$ \\
\hline $\begin{array}{l}\text { Pesquisa } \\
\text { Agrícola } \\
\text { Municipal }\end{array}$ & $\begin{array}{l}\text { Produção da lavoura temporária: Mel de abelha } \\
\text { (Quilogramas), Abacaxi (Mil frutos), Alho (Toneladas), } \\
\text { Amendoim (em casca) (Toneladas), Arroz (em casca) } \\
\text { (Toneladas), Aveia (em grão) (Toneladas), Batata-doce } \\
\text { (Toneladas), Batata-inglesa (Toneladas), Cana-de-açúcar } \\
\text { (Toneladas), Cebola (Toneladas), Centeio (em grão) } \\
\text { (Toneladas), Cevada (em grão) (Toneladas), Ervilha (em } \\
\text { grão) (Toneladas), Fava (em grão) (Toneladas), Feijão (em } \\
\text { grão) (Toneladas), Girassol (em grão) (Toneladas), Linho } \\
\text { (semente) (Toneladas), Mandioca (Toneladas), Melancia } \\
\text { (Toneladas), Melão (Toneladas), Milho (em grão) } \\
\text { (Toneladas), Soja (em grão) (Toneladas), Sorgo (em grão) } \\
\text { (Toneladas), Tomate (Toneladas), Trigo (em grão) } \\
\text { (Toneladas), Triticale (em grão) (Toneladas) }\end{array}$ & $\begin{array}{l}\text { Quantidade } \\
\text { produzida de } \\
\text { gêneros } \\
\text { alimentares }\end{array}$ \\
\hline $\begin{array}{l}\text { Pesquisa } \\
\text { Agrícola } \\
\text { Municipal }\end{array}$ & $\begin{array}{l}\text { Produção da lavoura permanente: Triticale (em grão) } \\
\text { (Toneladas), Abacate (Toneladas), Azeitona (Toneladas), } \\
\text { Banana (cacho) (Toneladas), Cacau (em amêndoa) } \\
\text { (Toneladas), Café (em grão) (Toneladas), Caqui (Toneladas), } \\
\text { Castanha de caju (Toneladas), Coco-da-baía (Mil frutos) } \\
\text { Dendê (cacho de coco) (Toneladas), Erva-mate (folha verde) } \\
\text { (Toneladas), Figo (Toneladas), Goiaba (Toneladas), Guaraná } \\
\text { (semente) (Toneladas), Laranja (Toneladas), Limão } \\
\text { (Toneladas), Maçã (Toneladas), Mamão (Toneladas), Manga } \\
\text { (Toneladas), Maracujá (Toneladas), Marmelo (Toneladas), } \\
\text { Noz (fruto seco) (Toneladas), Palmito (Toneladas), Pera } \\
\text { (Toneladas), Pêssego (Toneladas), Pimenta-do-reino } \\
\text { (Toneladas), Tangerina (Toneladas), Tungue (fruto seco) } \\
\text { (Toneladas), Urucum (semente) (Toneladas), Uva (Toneladas) }\end{array}$ & $\begin{array}{l}\text { Quantidade } \\
\text { produzida de } \\
\text { gêneros } \\
\text { alimentares }\end{array}$ \\
\hline $\begin{array}{l}\text { Censo } \\
\text { Agropecuário }\end{array}$ & $\begin{array}{l}\text { Produção na agricultura familiar, não familiar e total: } \\
\text { Produtos da horticultura em definição }\end{array}$ & $\begin{array}{l}\text { Quantidade } \\
\text { produzida de } \\
\text { gêneros } \\
\text { alimentares }\end{array}$ \\
\hline
\end{tabular}


3.2 Indicador 2 - Percentual da área ocupada pela agricultura familiar em relação à área total de produção

Para o indicador 2, foram selecionados os dados citados no Quadro 02, contemplando dados de área de produção e número de estabelecimentos agropecuários. Com os dados disponíveis será possível calcular o indicador agregado de percentual da área ocupada pela agricultura familiar nos municípios, conforme indicado pela CAISAN. Além dos dados de área, foram incluídos nesse indicador informações de número de estabelecimentos agropecuários, contribuindo para o maior detalhamento da distribuição dos produtores de alimentos nos municípios do Rio Grande do Sul. 
Quadro 02 - Dados selecionados para o Indicador 2, da Dimensão I

\begin{tabular}{|c|c|c|}
\hline Fonte & Dados selecionados & $\begin{array}{c}\text { Variável } \\
\text { selecionada }\end{array}$ \\
\hline $\begin{array}{l}\text { Censo } \\
\text { Agropecuário }\end{array}$ & $\begin{array}{l}\text { Área dos estabelecimentos agropecuários na agricultura } \\
\text { familiar, não-familiar e total }\end{array}$ & Área em hectares \\
\hline $\begin{array}{l}\text { Censo } \\
\text { Agropecuário }\end{array}$ & $\begin{array}{l}\text { Estabelecimentos agropecuários na agricultura familiar, não- } \\
\text { familiar e total }\end{array}$ & $\begin{array}{l}\text { Número de } \\
\text { estabelecimentos } \\
\text { agropecuários }\end{array}$ \\
\hline $\begin{array}{l}\text { Censo } \\
\text { Agropecuário }\end{array}$ & $\begin{array}{l}\text { Estabelecimentos agropecuários com lavouras e pastagens na } \\
\text { agricultura familiar, não-familiar e total }\end{array}$ & $\begin{array}{l}\text { Área em hectares } \\
\text { de lavouras e } \\
\text { pastagens }\end{array}$ \\
\hline $\begin{array}{l}\text { Censo } \\
\text { Agropecuário }\end{array}$ & $\begin{array}{l}\text { Estabelecimentos agropecuários com pecuária de corte na } \\
\text { agricultura familiar, não-familiar e total }\end{array}$ & Área em hectares \\
\hline
\end{tabular}

3.3 Indicador 3 - Percentual da área ocupada por grupo dos principais produtos dirigidos para alimentação no mercado interno em relação à área total

A seleção e análise dos dados para o indicador 3 segue o mesmo contexto do indicador 1, contemplando dados que poderão ser agregados pelas categorias de agricultura familiar e não-familiar, e outros que não possibilitam esta classificação. Todavia, como o indicador sugerido pela CAISAN não delimita a necessidade dessa classificação adicional, todos os dados poderão ser utilizados para o cálculo do indicador, conforme a especificação da CAISAN. Os produtos selecionados referem-se a todos os gêneros alimentícios disponíveis nos bancos de dados consultados, divididos em lavoura permanente e temporária.

Quadro 03. Dados selecionados para o Indicador 3, da Dimensão I

\begin{tabular}{|l|l|l|}
\hline \multicolumn{1}{|c|}{ Fonte } & \multicolumn{1}{c|}{ Dados selecionados } & \multicolumn{1}{c|}{$\begin{array}{c}\text { Variável } \\
\text { selecionada }\end{array}$} \\
\hline $\begin{array}{l}\text { Censo } \\
\text { Agropecuário }\end{array}$ & $\begin{array}{l}\text { Produção da lavoura permanente na agricultura familiar, } \\
\text { não-familiar e total: Banana, Café arábica em grão (verde), } \\
\text { Café canephora (robusta, conilon) em grão (verde), Laranja, } \\
\text { Uva }\end{array}$ & $\begin{array}{l}\text { Área plantada em } \\
\text { hectares }\end{array}$ \\
\hline Censo & Produção da lavoura temporária na agricultura familiar, não- & Área plantada em \\
\hline
\end{tabular}


Angélica C. Siqueira, Daniela Wives, Tathiane M. Medeiros, Natany M. Schreiber, Leonardo X. Silva, Gabriela C. Souza

\begin{tabular}{|c|c|c|}
\hline Agropecuário & $\begin{array}{l}\text { familiar e total: Arroz em casca, Cana-de-açúcar, Cebola, } \\
\text { Feijão em grão, Mandioca (aipim, macaxeira), Milho em } \\
\text { grão, Soja em grão, Trigo em grão }\end{array}$ & hectares \\
\hline $\begin{array}{l}\text { Pesquisa } \\
\text { Agrícola } \\
\text { Municipal }\end{array}$ & $\begin{array}{l}\text { Área da lavoura temporária: Abacaxi (Mil frutos), Alho } \\
\text { (Toneladas), Amendoim (em casca) (Toneladas), Arroz (em } \\
\text { casca) (Toneladas), Aveia (em grão) (Toneladas), Batata-doce } \\
\text { (Toneladas), Batata-inglesa (Toneladas), Cana-de-açúcar } \\
\text { (Toneladas), Cebola (Toneladas), Centeio (em grão) } \\
\text { (Toneladas), Cevada (em grão) (Toneladas), Ervilha (em grão) } \\
\text { (Toneladas), Fava (em grão) (Toneladas), Feijão (em grão) } \\
\text { (Toneladas), Girassol (em grão) (Toneladas), Linho (semente) } \\
\text { (Toneladas), Mandioca (Toneladas), Melancia (Toneladas), } \\
\text { Melão (Toneladas), Milho (em grão) (Toneladas), Soja (em } \\
\text { grão) (Toneladas), Sorgo (em grão) (Toneladas), Tomate } \\
\text { (Toneladas), Trigo (em grão) (Toneladas), Triticale (em grão) } \\
\text { (Toneladas) }\end{array}$ & $\begin{array}{l}\text { Área plantada em } \\
\text { hectares }\end{array}$ \\
\hline
\end{tabular}


Continuação

\begin{tabular}{|c|c|c|}
\hline Fonte & Dados selecionados & $\begin{array}{l}\text { Variável } \\
\text { selecionada }\end{array}$ \\
\hline $\begin{array}{l}\text { Pesquisa } \\
\text { Agrícola } \\
\text { Municipal }\end{array}$ & $\begin{array}{l}\text { Área da lavoura permanente: Triticale (em grão) } \\
\text { (Toneladas) Abacate (Toneladas), Azeitona (Toneladas), } \\
\text { Banana (cacho) (Toneladas), Cacau (em amêndoa) } \\
\text { (Toneladas), Café (em grão) (Toneladas), Caqui } \\
\text { (Toneladas), Castanha de caju (Toneladas), Coco-da-baía } \\
\text { (Mil frutos), Dendê (cacho de coco) (Toneladas), Erva-mate } \\
\text { (folha verde)(Toneladas), Figo (Toneladas), Goiaba } \\
\text { (Toneladas), Guaraná (semente) (Toneladas), Laranja } \\
\text { (Toneladas), Limão (Toneladas), Maçã (Toneladas), Mamão } \\
\text { (Toneladas), Manga (Toneladas), Maracujá (Toneladas), } \\
\text { Noz (fruto seco) (Toneladas), Palmito (Toneladas), Pera } \\
\text { (Toneladas), Pêssego (Toneladas), Pimenta-do-reino } \\
\text { (Toneladas), Tangerina (Toneladas), Tungue (fruto seco) } \\
\text { (Toneladas), Urucum (semente) (Toneladas),Uva } \\
\text { (Toneladas) }\end{array}$ & $\begin{array}{l}\text { Área plantada } \\
\text { em hectares }\end{array}$ \\
\hline
\end{tabular}

3.4 Indicador 4 - Percentual da área sob utilização de sistemas sustentáveis de produção (Agricultura Orgânica + Plantio Direto)

Para o indicador 4, a CAISAN sugere como fonte de dados entidades públicas (Cadastro Nacional da Produção Orgânica) e privadas (APDC). Para o OBSSAN, até o momento foram selecionados dados do Censo Agropecuário, contemplando informações sobre a forma de cultivo que podem refletir os resultados esperados para este indicador. Apenas um dos dados selecionados contém informações sobre área de cultivo, de forma que serão coletadas as informações de número de estabelecimentos para os demais dados. Entende-se que tais informações poderão responder, em parte, a questão levantada neste indicador, compondo o cenário da utilização de manejos sustentáveis na produção de alimentos dos municípios.

\section{Quadro 04. Dados selecionados para o Indicador 4, da Dimensão I}

\begin{tabular}{|l|l|l|}
\hline Fonte & Dados selecionados & $\begin{array}{l}\text { Variável } \\
\text { selecionada }\end{array}$ \\
\hline $\begin{array}{l}\text { Censo } \\
\text { Agropecuário }\end{array}$ & $\begin{array}{l}\text { Estabelecimentos agropecuários na agricultura familiar, não- } \\
\text { familiar e total: tipos de utilização de terras em definição }\end{array}$ & $\begin{array}{l}\text { Área em hectares } \\
\text { e número de } \\
\text { estabelecimentos }\end{array}$ \\
\hline
\end{tabular}


Angélica C. Siqueira, Daniela Wives, Tathiane M. Medeiros, Natany M. Schreiber, Leonardo X. Silva, Gabriela C. Souza

\begin{tabular}{|l|l|l|}
\hline & & agropecuários \\
\hline $\begin{array}{l}\text { Censo } \\
\text { Agropecuário }\end{array}$ & $\begin{array}{l}\text { Estabelecimentos agropecuários na agricultura familiar, não- } \\
\text { familiar e total: tipos de prática agrícola em definição }\end{array}$ & $\begin{array}{l}\text { Número de } \\
\text { estabelecimentos } \\
\text { agropecuários }\end{array}$ \\
\hline $\begin{array}{l}\text { Censo } \\
\text { Agropecuário }\end{array}$ & $\begin{array}{l}\text { Estabelecimentos agropecuários na agricultura familiar, não-- } \\
\text { familiar e total: tipos de uso de agrotóxicos em definição }\end{array}$ & $\begin{array}{l}\text { Número de } \\
\text { estabelecimentos } \\
\text { agropecuários }\end{array}$ \\
\hline $\begin{array}{l}\text { Censo } \\
\text { Agropecuário }\end{array}$ & $\begin{array}{l}\text { Estabelecimentos agropecuários na agricultura familiar, não- } \\
\text { familiar e total: tipos de alternativas para controle de pragas } \\
\text { e/ou doenças em vegetais em definição }\end{array}$ & $\begin{array}{l}\text { Número de } \\
\text { estabelecimentos } \\
\text { agropecuários }\end{array}$ \\
\hline $\begin{array}{l}\text { Censo } \\
\text { Agropecuário }\end{array}$ & $\begin{array}{l}\text { Estabelecimentos agropecuários na agricultura familiar, não- } \\
\text { familiar e total: tipos de uso de irrigação em definição }\end{array}$ & $\begin{array}{l}\text { Número de } \\
\text { estabelecimentos } \\
\text { agropecuários }\end{array}$ \\
\hline
\end{tabular}

4 Discussão dos Indicadores

No Brasil, a elaboração do Plano Nacional de SAN e das políticas de SAN assentam-se na premissa de uma busca efetiva pela incorporação do DHAA. Assim, desenvolver e refinar instrumentos capazes de monitorar e analisar como esse direito está sendo realmente incorporado por tais políticas e programas é cada vez mais imprescindível.

O DHAA é indispensável para o bem estar das sociedades, sejam rurais ou urbanas. As normas internacionais reconhecem o direito de todos à alimentação adequada e o direito fundamental de toda pessoa a estar livre da fome, como pré-requisitos para a realização de outros direitos humanos (BURITY et al., 2010). Considerando tal afirmação, o DHAA decorre da real incorporação de várias estratégias de desenvolvimento com uma abordagem multinível e multiescalar em que haja a construção de políticas e programas que atuem efetivamente numa perspectiva abrangente e que considerem a SAN como um caminho eficaz para atender esse que é um dos direitos mais básicos do ser humano.

Considerando o caso brasileiro, há uma insuficiência de medidas que munam a mensuração apropriada do DHAA. Ressalta-se que é imperativo considerar elementos que investiguem como, onde e quanto se tem avançado em concretizá-lo. Nesse sentido destaca-se a pertinência de estudos, como os do OBSSAN-RS, a cerca do instrumento de monitoramento proposto no âmbito do PLANSAN. E pode-se afirmar 
por meio da análise aqui apresentada que, na dimensão I, os seis indicadores propostos atentem, com dados disponíveis, apenas uma escala macroespacial. Assim, ressalta-se que na agregação territorial municipal, as informações disponíveis nos bancos de dados oficiais são insuficientes para gerar os resultados solicitados nos indicadores. Medeiros et al. (2014) não consideraram disponíveis os indicadores da dimensão 1 do PLANSAN. Todavia, observou-se que os dados aferidos em pesquisas do IBGE - incluindo o Censo Agropecuário já indicado no PLANSAN 2012/2015 - contém informações importantes para a compreensão do contexto da produção de alimentos dos municípios do estado. Dessa forma, limitando a análise pelos indicadores propostos pela CAISAN, pode-se concluir que não é possível aferir os resultados dos indicadores 1 e 4, mas há dados suficientes para análise dos indicadores 2 e 3 .

Porém, como já levantado anteriormente, entende-se que há outros dados que podem ser considerados para a análise dos indicadores 1 e 4 em termos municipais, apesar de não responderem exatamente aos indicadores propostos no PLANSAN. De forma que torna-se viável, contando apenas com dados já aferidos em pesquisas do IBGE, avaliar a Dimensão de Produção de Alimentos nos municípios do Rio Grande do Sul, desde que adaptando as metodologias indicadas pela CAISAN. Além disso, neste trabalho foram sugeridos dados adicionais para análise de cada indicador, ampliando as possibilidades de pesquisa e avaliação da SAN nos municípios do estado, os quais podem ser considerados para futuras análises da Dimensão I em outras regiões do país.

Não se tem a pretensão de fornecer elementos que possam constituir um "mapa do caminho", porém, crê-se na força de uma preceptiva de desenvolvimento regional subsidiada por instrumentos de gestão social como o OBSSAN. Entende-se a possibilidade de um olhar compartilhado, de construção institucional e político, de baixo para cima, por meio de uma visão profunda das realidades locais e regionais. Além disso, o fomento de ferramentas de avaliação e monitoramento permite observar as potencialidades e lacunas de determinadas políticas e programas, como por exemplo, a potencialidade das recentes políticas de segurança alimentar e nutricional, voltadas aos povos indígenas e comunidades tradicionais. 


\section{Considerações finais}

Nesse contexto, conclui-se que os indicadores propostos pela CAISAN para a Dimensão I alcançam, em parte, as necessidades para o monitoramento da SAN no que respeita a municípios.

Os quatro indicadores que compõem a dimensão produção de alimentos destacam a importância da agricultura familiar e de sistemas sustentáveis na produção de alimentos do município. O destaque para a Agricultura Familiar na dimensão 1 busca 0 monitoramento do fortalecimento da agricultura familiar, a qual é objetivo de políticas públicas desde 1996, com o início do Programa Nacional de Fortalecimento da Agricultura Familiar - PRONAF no Governo de Fernando Henrique Cardoso. Destaca-se que a avaliação a partir do grupo social carece de complementação dos povos indígenas e comunidades tradicionais, os quais são grupos que historicamente desenvolvem sistemas sustentáveis. Entretanto, incluir povos indígenas e comunidades tradicionais no mesmo indicador provocaria uma diluição nos resultados dos esforços sobre a agricultura familiar, tendo em vista que políticas públicas para esses coletivos tradicionais são muito recentes e o volume de recursos bastante escassos. Como forma de minimizar essa situação, sugere-se a criação de indicadores de produção específicos para esses grupos, bem como a inclusão de indicadores que contemplem o autoconsumo, prática comum em todos esses grupos.

A dimensão 1, ao atrelar grupos sociais a práticas produtivas sustentáveis, também tende a imprimir uma análise enviesada, pois o grupo social per se não é garantia do desenvolvimento efetivo de um sistema de produção sustentável. Da mesma forma, a avaliação com base no produto sustentável também, não obrigatoriamente, está atrelada a um determinado grupo social. Com base no cruzamento desses indicadores, percebe-se que o monitoramento está buscando precisar a produção sustentável por grupos sociais reconhecidos no cenário brasileiro por suas práticas sustentáveis. Entretanto, há de se considerar as imprecisões geradas ao assumir tais pressupostos. Nesse caso, sugere-se imprimir maior relevância à análise dos indicadores separadamente. 
Soma-se ao já observado acima, que trata-se de um objeto e um tema de grande abrangência, ou seja, a promoção da garantia doDHAA. Assim, assume-se que esse direito também engloba a promoção de uma nova política de ordenamento territorial, fortalecimento da agricultura familiar e populações tradicionais, de políticas de abastecimento, de estímulo a estilos de agricultura de base ecológica e o fortalecimento das práticas e saberes tradicionais

Dessa forma, ressalta-se a importância dos esforços elencados no escopo do OBSSAN-RS, pois entende-se que na perspectiva da promoção dos direitos humanos, o processo como é feito é tão importante quanto o resultado. De tal modo, torna-se fundamental a internalização e disseminação de conhecimentos sobre o conceito, os princípios e as dimensões desse direito humano fundamental. Nesse sentido, nos esforços despendidos no OBSSAN-RS, uma questão chave apoia-se na compreensão que o direito a uma alimentação adequada ultrapassa pensamentos e ações com implicações paternalistas, assistencialistas, discriminatórias e autoritárias. Nesse sentido, defende-se que a efetivação e a implantação de programas e políticas públicas de SAN sejam sempre amplamente analisados e debatidos por um processo democrático, participativo, inclusivo, que respeite as diferenças e diversidades entre os grupos sociais. Mas, sobretudo, que seu monitoramento seja garantido. Isso aponta claramente para a necessidade de espaços como o OBSSAN-RS. Ambiente no qual essas reflexões estão sendo organizadas no intuito de fortalecer a integração entre os diferentes campos de saber, e, portanto, a análise multidisciplinar e articulação social das Políticas Públicas de SAN.

Apesar do termo "observatório" sugerir, inicialmente, uma atitude de distanciamento, percebe-se que esse instrumento toma postura ativa sobre a realidade observada. Tanto por contribuir para a diminuição da assimetria de informações (e poder) quanto porque sabe-se que o ser humano não observa per se, mas reflete sobre as informações adquiridas, modificando sua posição e atuação sobre o ambiente. Dessa forma, entende-se que os observatórios também visam à transformação de sua área, e a abordagem articulada às demandas sociais, portanto, assume uma perspectiva de conhecimento que transcende o acadêmico, gerando intervenções práticas na realidade.

Considera-se, ainda, que a alocação de observatórios em 
Universidades torna-se estratégico ao passo que proporciona a abertura de um amplo campo de pesquisa e de estreitamento de seus vínculos com a sociedade por meio da extensão. Dessa forma, levando em conta o papel das universidades como centros de produção de conhecimento, os observatórios agregam capacidade técnica à sociedade civil, proporcionando maior legitimidade e abrindo espaços (arenas políticas) para a interação entre os atores sociais envolvidos, desde as comunidades até os gestores públicos. E, assim, pode contribuir para materializar o compromisso político e cumprir a função social da universidade.

Todavia, tem-se ainda um importante caminho a trilhar, desenvolvendo metodologias de trabalho inovadoras, que possam viabilizar a efetiva troca de saberes entre os diferentes atores envolvidos, a apropriação dos conhecimentos gerados, e seu retorno para a transformação da realidade observada, subsidiando ações de assistência técnica, gestão, Educação Alimentar e Nutricional e, assim, contribuir para o aprimoramento dos diálogos institucionais e da SAN como um todo no Rio Grande do Sul, abrindo caminhos para o fortalecimento da gestão democrática nas políticas de SAN, da cidadania e, por conseguinte, do DHAA.

\section{Agradecimento}

Os autores agradecem ao CNPQ pela bolsa PIBIC da primeira autora, à CAPES pela bolsa de mestrado da quarta autora e a bolsa PNPD da segunda autora, ao MEC/SESU pelo financiamento do Projeto do OBSSAN-RS e ao Conselho Estadual de Segurança Alimentar e Nutricional do Rio Grande do Sul, na pessoa do seu coordenador Miguel Montaña, pelo apoio à pesquisa.

\section{REFERÊNCIAS}

BRASIL. Constituição Federal. Brasília: Senado Federal, 1988.

BRASIL. Decreto Federal 1946, de 28 de junho de 1996. Cria o Programa Nacional de Fortalecimento da Agricultura Familiar - PRONAF, e dá 
outras providências. Brasilia, DF, 1996.

BRASIL. Congresso Nacional. Lei 11.346, de 15 de setembro de 2006. Lei Orgânica de Segurança Alimentar e Nutricional (LOSAN). Brasília, DF, 2006.

BRASIL. Decreto $n^{0}$ 6.040, de 7 de fevereiro de 2007. Institui a Política Nacional de Desenvolvimento Sustentável dos Povos e Comunidades Tradicionais. Disponível em: $<$ http://www.planalto.gov.br/ccivil_03/_ato20072010/2007/decreto/d6040.htm>. Acesso em: 07 jan. 2013.

BRASIL. Decreto $n^{\circ} 7.272$, de 25 de agosto de 2010. Institui a Política Nacional de Segurança Alimentar e Nutricional. Brasília, 2010.

BRASIL. Câmara Interministerial de Segurança Alimentar e Nutricional CAISAN. Plano Nacional de Segurança Alimentar e Nutricional: 2012/2015. Brasília, DF, 2011.

BRASIL. Decreto Federal $n^{\circ} 7.747$, de 5 de junho de 2012. Institui a Política Nacional de Gestão Territorial e Ambiental de Terras Indígenas PNGATI, e dá outras providências. Brasília, 2012.

BURITY, V.; FRANCESCHINI, T.; VALENTE, F.; RECINE, E.; LEÃO, M.; CARVALHO, M. F. Direito Humana à Alimentação Adequada no Contexto da Segurança Alimentar e Nutricional. Brasília: 2010. 204 p.

CONSELHO NACIONAL DE SEGURANÇA ALIMENTAR E NUTRICIONAL CONSEA. IV Conferência de Segurança Alimentar e Nutricional. Documento de Referência. Brasília, DF, 2011.

MEDEIROS, T.M., XAVIER, L., COELHO DE SOUZA, G. Segurança Alimentar e Nutricional no meio rural do município de Gravataí: uma discussão sobre indicadores de diagnósticos locais e de nível municipal. In: XV Simpósio Internacional IHU. Anais... Alimento e Nutrição no contexto dos Objetivos de Desenvolvimento do Milênio", submetido, 2014. 
Organização das Nações Unidas. Declaração Universal dos Direitos Humanos. Disponível em:

$<$ http://www.direitoshumanos.usp.br/counter/Onu/Sist_glob_trat/texto /texto_1.html>. Acesso em: 16 nov. 2011.

TELES, J., MESQUITA, D., CEPEDA, A. Por um Sistema Nacional de Indicadores de Direitos Humanos. Revista Brasileira de Monitoramente e Avaliação, 2:168-171, 2011.

UNICEF. Os objetivos de desenvolvimento do milênio, 2014. Disponível em:

$<$ http://www.unicef.pt/docs/os_objectivos_de_desenvolvimento_do_mil enio.pdf>. Acesso em: 07 abr. 2014.

Submetido em 24/08/2014

Aprovado em 13/01/2016

Sobre os autores

Angélica Cristina da Siqueira

Pesquisadora do Observatório Socioambiental em Segurança Alimentar e Nutricional UFRGS.

E-mail: ange.siq@gmail.com

\section{Daniela Garcez Wives}

Geógrafa. Pós-Doutoranda do Programa de Pós-Graduação em Desenvolvimento Rural. Pesquisadora do Núcleo de Estudos em Segurança Alimentar e Nutricional NESAN/UFRGS.

E-mail: garcezd@gmail.com

Tathiane Muriel Medeiros

Mestre em Desenvolvimento Rural - UFRGS.

E-mail: .tathi_ufrgs@yahoo.com.br

\section{Natany Meregalli Schreiber}

Graduanda em Agronomia. Bolsista de pesquisa do Observatório Socioambiental em Segurança Alimentar e Nutricional e do Núcleo de Estudos e Pesquisas em Segurança Alimentar e Nutricional - UFRGS. 
Observatório Socioambiental em Segurança Alimentar e Nutricional: análise dos indicadores...

E-mail: natanyschreiber@gmail.com

\section{Leonardo Xavier da Silva}

Economista. Docente da Faculdade de Ciências Econômicas e do Programa de PósGraduação em Desenvolvimento Rural PGDR/UFRGS.

E-mail: leonardo.xavier@ufrgs.br

Gabriela Coelho-de-Souza

Bióloga. Docente da Faculdade de Ciências Econômicas e do Programa de PósGraduação em Desenvolvimento Rural PGDR/UFRGS. Coordenadora do NESAN.

E-mail: gabrielacoelhoddesouza@pq.cnpq.br 